\title{
The Relationship between Suicide Incidents in Slovakia and the Czech Republic and Heliophysical Parameters: Empirical Results
}

\section{Kancírová $\mathbf{M}^{1,2}$ and Kudela $\mathbf{K}^{3 *}$}

${ }^{1}$ Department of Mathematics and Physics, The University of Security Management, Kukučínova 17, 04001 Košice, Slovakia

${ }^{2}$ Faculty of Science, P.J. Šafárik University, Košice, Slovakia

${ }^{3}$ IEP SAS, Watsonova 47, 04001 Košice, Slovakia

\begin{abstract}
Suicide has been attributed to social and economic factors but changes in solar radiation and geomagnetic activities may contribute to the frequency and the seasonal pattern of suicides. We studied the relationship between suicide episodes in Slovakia and the Czech Republic and parameters of solar, heliospheric and geomagnetic activity during the period of 1982 to 2011 . Along with checking the linear correlation between the suicide event number and the physical parameters of outer space on yearly basis, the contribution of the harmonic component with periodicity near that of the heliophysical chracteristics was examined and optimum frequency of such contribution was estimated used detrended time series. The results show a weak effect of solar and geomagnertic activity variations on the suicide incidents in the given data set. Higher statistics and better temporal resolution of the data is needed to obtain more conclusive results.
\end{abstract}

Keywords: Suicide incidents; Cosmic ray intensity; Sunspot number; Ap-index; $10.7 \mathrm{~cm}$ radio flux

\section{Introduction}

The possible impact of solar, cosmic rays and geomagnetic activity has become the subject of many research studies. Several studies have been made to investigate space weather hazards and their impact on human cardio-health state parameters. It was shown that geomagnetic activity (GMA) and cosmic ray intensity (CRI) variations could be considered as one of the indicators of space weather, which play a role in regulating external factors in human homeostasis, particularly, cardio - vascular health state [1-4]. Moreover, CRI decreases together with GMA index variations have been analyzed with regard to heart rate (HR) and beat-to-beat heart rate (RR) interval variations. RR intervals and HR showed opposite correlations which proved the reliability of the conducted measurements. The most interesting and common result that has been revealed is that HR and RR interval variations appear to be connected to geomagnetic disturbances and CRI variations. The effects are more pronounced for high levels of GMA and strong CRI decreases [3].

Verma [5] reported that solar activity parameters (sunspot numbers, solar flare index, coronal index, CRI) are significantly connected with yearly suicide incidence during the period from 1989 to 2011. The Cornelissen et al. paper [6] on exploring any non-photic as well as photic components in the spectrum of the incidence pattern of suicides, reports the gender differences in Minnesota, USA, and Australia, notably in the para-annual range of the spectrum. The links between solar activity and cardiovascular diseases have been studied too [7-9].

The paper by Palmer et al. [10] summarizes the research undertaken in the field of heliobiology, focusing on the effect of variations of geomagnetic activity on human cardiovascular health. The three conclusions are that geomagnetic effects are more pronounced at higher magnetic latitudes; that extremely high as well as extremely low values of geomagnetic activity seem to have adverse health effects and that a subset of the population (10-15\%) is predisposed to adverse health effects due to geomagnetic variations. Results of the paper by Gordon and Berk [11] support the hypothesis that a geomagnetic storm activity is associated with suicide rates, in both male and female population in South Africa.

The aim of this study is to explore possible links between suicide incidence in Slovakia and in Czech Republic and heliophysical parameters on yearly basis.

\section{Data and Analysis}

Time series datasets consist of annual male, female, total and rate suicide incidents (per 100,000 population) in Slovakia and in the Czech Republic. The data of suicide incidence in Slovakia has been taken from the National Health Information Center (NHIC) [12,13], the data in the Czech Republic has been taken from the Czech Statistical Office (CZSO) [14]. Data are plotted in Figure 1. Monthly means of sunspot number (SSN) data were obtained from NASA, available at http://solarscience.msfc.nasa.gov/greenwch/spot_num.txt and average annual data computed. Daily mean of Ap-index data were taken from the British Geological Survey (http://www.geomag.bgs.ac.uk/data service/data/magnetic_indices/apindex.html) and average annual data computed. Monthly averages of Solar $10.7 \mathrm{~cm}$ radio flux data were obtained from the NOAA, currently also online (ftp://ftp.geolab.nrcan. gc.ca/data/solar_flux/monthly_averages/solflux_monthly_average.txt) and average annual data computed. Hourly pressure corrected data of the CRI were obtained from the online data base of Lomnický štít Neutron monitor (http://neutronmonitor.ta3.sk/ ). Data are displayed in Figure 2.

As a first step we computed the linear correlation coefficients between suicide event occurrence and the outer space physical data

*Corresponding author: Kudela K, IEP SAS, Watsonova 47, 04001 Košice, Slovakia, Tel: 4215562221 Email: kkudela@kosice.upjs.sk

Received June 11, 2014; Accepted July 04, 2014; Published July 06, 2014

Citation: Kancírová M, Kudela K (2014) The Relationship between Suicide Incidents in Slovakia and the Czech Republic and Heliophysical Parameters: Empirical Results. Astrobiol Outreach 2: 116. doi: 10.4172/2332-2519.1000116

Copyright: @ 2014 Kancírová M, et al. This is an open-access article distributed under the terms of the Creative Commons Attribution License, which permits unrestricted use, distribution, and reproduction in any medium, provided the original author and source are credited. 
Citation: Kancírová M, Kudela K (2014) The Relationship between Suicide Incidents in Slovakia and the Czech Republic and Heliophysical Parameters: Empirical Results. Astrobiol Outreach 2: 116. doi: 10.4172/2332-2519.1000116

Page 2 of 5

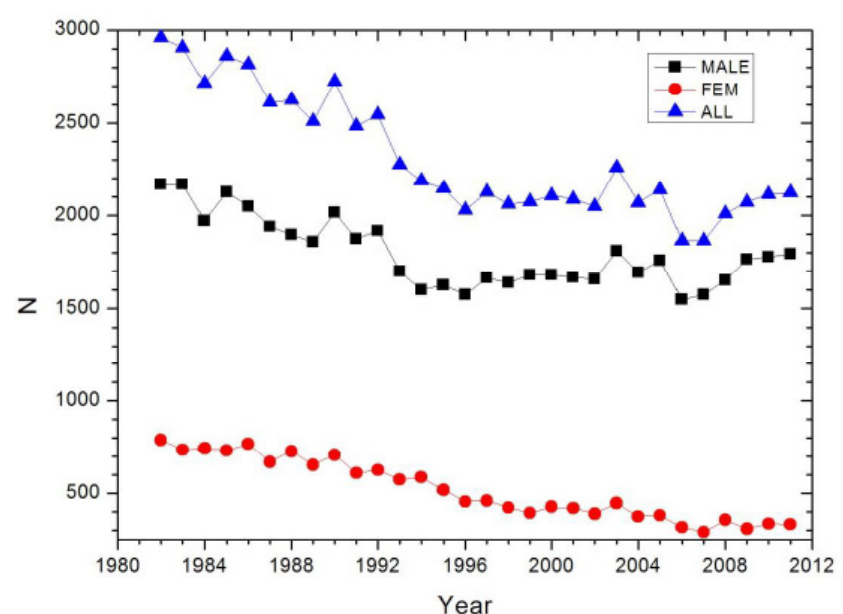

Figure 1: The annual variation of suicide incidents during the period from 1982 to 2011 (male: black, female: red, total: blue). The annual incidence of suicides in Slovakia and the Czech Republic (data for the period from 1982 to 2011 are the sum of the number of suicide incidents in Slovakia and the Czech Republic).
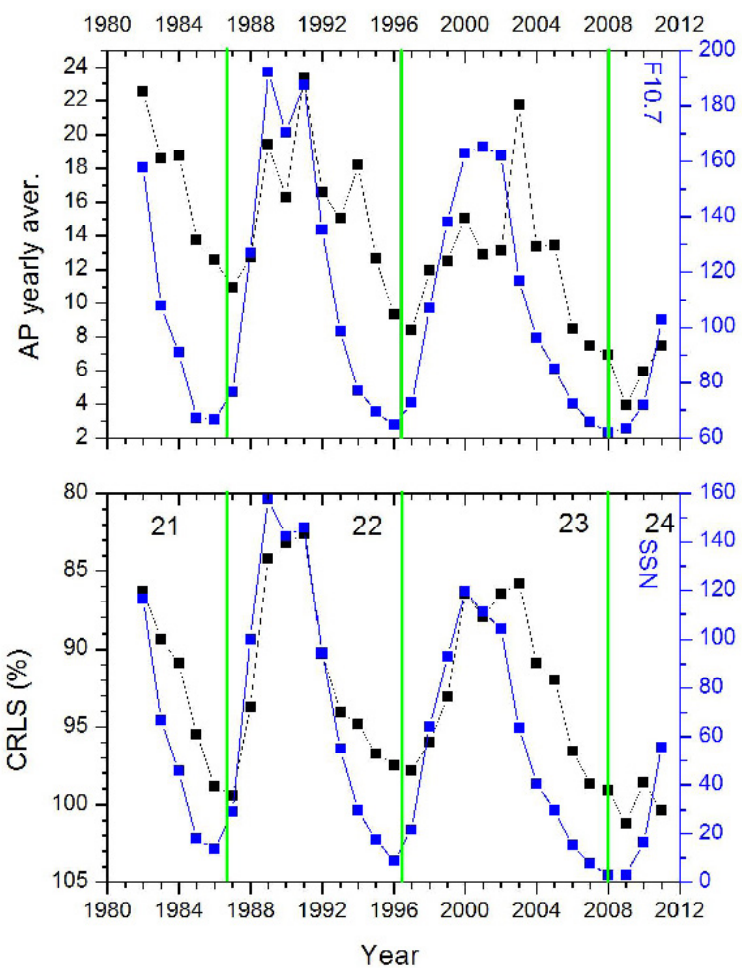

Figure 2: Yearly means of solar activity indices (Sunspot numbers and radio wave solar emission at $10.7 \mathrm{~cm}$ wavelength which provides mutual correlation almost unity on yearly basis), geomagnetic activity Ap-index and Cosmic ray intensity at Lomnický štít. Solar cycle numbers are marked. CRLS (\%) definition is at http://neutronmonitor.ta3.sk/description.php .

(Table 1). The statistical methods of computation the linear correlations used here are described in the book by Kenny [15].

Higher $\mathrm{r}$ is obtained in all three subsets for the correlation with geomagnetic activity index Ap. Recently, paper by Tada et al. [16] analyzing large sample of the data over 1999-2010 in Japan, found that for males the number of suicides was associated with the monthly mean geomagnetic activity $\mathrm{K}$ index, but for females it was not the case. Recently, the importance of the background magnetic field for suicides was stressed in paper by Nishimura et al. [17]. A hypothesis that stronger geomagnetic field affects the number of male suicides, based on detailed dtstidticsl study in various regions of Japan, was presented. In our case (lower statistics and yearly time resolution) we are unable to infer the differences for males and females. For Ap the significance of the differences between two correlation coefficients provides rather high value $\mathrm{p}=0.20$.

Although there is a high degree of correlation between the solar activity indices, cosmic rays and geomagnetic activity, the differences are also seen in Figure 2 at least in two aspects: a. cosmic ray delay (hysteresis in the declining phases of solar activity, especially in the cycles 21 and 23 - due to drifts of cosmic rays and different polarity of the heliospheric magnetic field - since CR reflect the "global" state of the heliosphere, not only its inner region), b. Ap local maxima as in year 2003 (may be reflecting the extreme geomagnetic events in October November of that year) is not clearly seen in the solar activity yearly averages. Thus the linear multiple regression of N ALL with respect to variables AP, CRI and SSN has been performed (Figure 3).

Assuming low number of points (30), the statistical significance (Table 1 and Figure 3 ) is not high. Geomagnetic activity index provides the best correlation (Table 1). The linear multiregression method provides higher correlation coefficients. While solar (SSN, F10.7), heliophysical (CRI) and geomagnetic (Ap) indices show almost

\begin{tabular}{|c|c|c|c|c|c|}
\hline & Male & Female & Total & $\begin{array}{c}\text { N/10^5 Number per 10^5 } \\
\text { population }\end{array}$ & $\begin{array}{c}\mathbf{p} \\
\mathbf{( N / 1 0 \wedge 5 )}\end{array}$ \\
\hline CRI & -0.303 & -0.346 & -0.340 & -0.335 & 0.07 \\
\hline SSN & 0.290 & 0.344 & 0.332 & 0.336 & 0.07 \\
\hline Ap-index & 0.487 & 0.642 & 0.589 & 0.607 & 0.0004 \\
\hline F10.7 & 0.226 & 0.251 & 0.250 & 0.255 & 0.17 \\
\hline
\end{tabular}

Table 1: Linear correlation coefficients $(r)$ between suicide incidents and cosmic ray intensity, sunspot numbers, Ap-index and $10.7 \mathrm{~cm}$ radio flux. Using $t=r /$ $\operatorname{sqrt}\left[\left(1-r^{2}\right) /(n-2)\right]$ and testing the null hypothesis that the observed value comes from a population in which $\rho=0$ we obtain $p$ (in the last column for the values Per100,000 population, using the calculator available at http://vassarstats.net/rsig. $\mathrm{html})$.
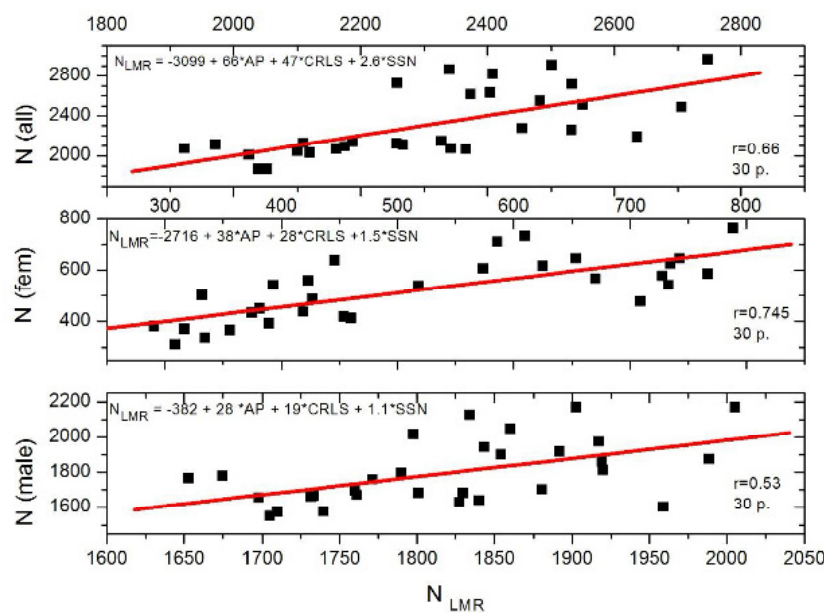

Figure 3: Linear multiregression between $\mathrm{N}$ and Ap, SSN, CRLS, respectively $\left(\mathrm{N}_{\mathrm{MR}}\right)$. Forms of the fits and correlation coefficients of the approximations are shown too. 
Citation: Kancírová M, Kudela K (2014) The Relationship between Suicide Incidents in Slovakia and the Czech Republic and Heliophysical Parameters: Empirical Results. Astrobiol Outreach 2: 116. doi: 10.4172/2332-2519.1000116
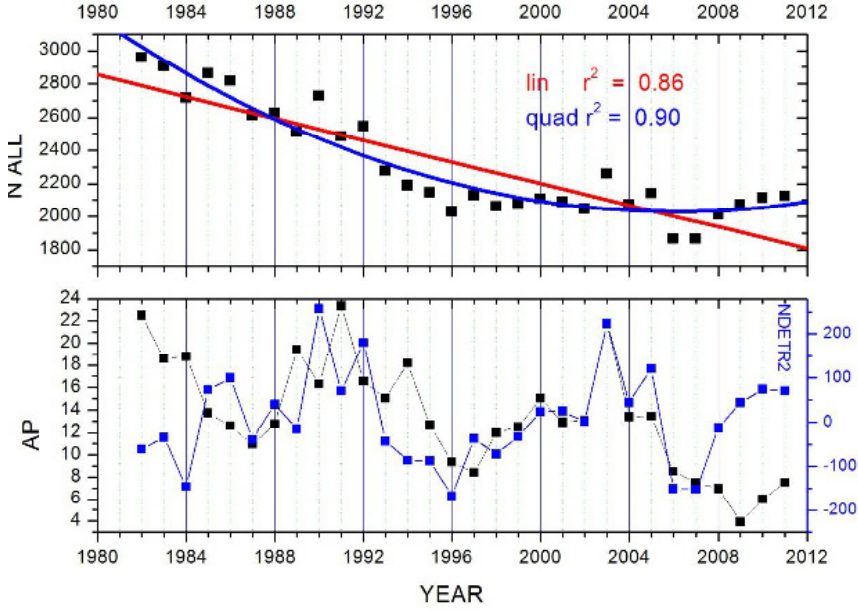

Figure 4: Upper panel: total number of incidences (N ALL) and time detrending. Values $r^{2}$ indicate quality of fits. Lower panel: quadratically detrended NDETR2 (scale to right) and Ap (scale to left).

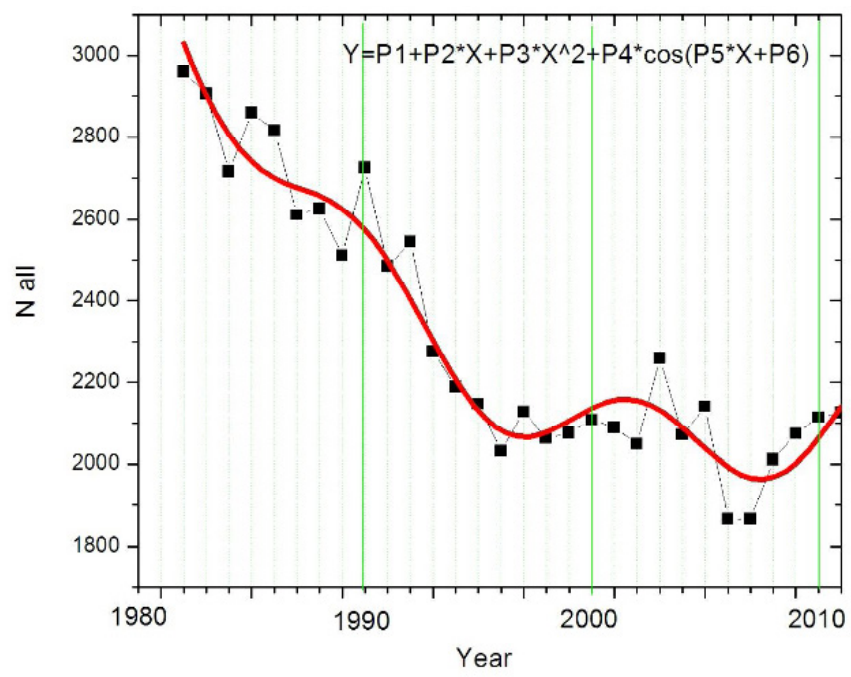

Figure 5: Best fit of the form given in the corner of figure. P1-P3 are taken the same as in Figure 4; $P 4=87 \pm 23 ; P 5=0.53 \pm 0.03 ; P 6=1.59 \pm 0.26$. The estimate of contribution of the cyclicity revealed with respect to detrended data is (according to ratio of the amplitude of the harmonic to the characteristic value of $\mathrm{N}$ ) of the order of $2-3 \%$.

stationary time series, the suicide event number(s) time profiles include clearly the long term trend(s). To detrend the time series we used linear and quadratic approximation illustrated in Figure 4.

Detrending the time series of suicides incidence (NDETR2) shows some similarities with Ap (and also with CR and SSN too). The time detrending itself leads to rather good fit. Thus there is present probably a significant longer time component of the variation of incidences than only that corresponding to cyclicities of solar, geomagnetic and cosmic ray intensities (most pronounced $\sim 11$ year), which is not related to heliophysical variability.

\section{Discussion}

To identify a possible contribution of the harmonic signal(s) with the frequency around that of the solar cycle activity (and related geomagnetic and cosmic ray intensities) to the profile $\mathrm{N}(\mathrm{t})$, we performed the nonlinear curve fitting of the form $\mathrm{N}(\mathrm{t})=\mathrm{P} 1+\mathrm{P} 2^{*} \mathrm{t}$ $+\mathrm{P} 3^{\star} \mathrm{t}^{2}+\mathrm{P} 4^{*} \cos \left(\mathrm{P} 5^{\star} t+\mathrm{P} 6\right)$, where $\mathrm{t}$ is time in years from $1982, \mathrm{P} 5=2.0 / \mathrm{T}$, $\mathrm{T}$ is length of the cycle, and P1- P3 are taken from the fit in Figure 4 upper part. Assuming P4, P5 and P6 as variables, the best fit according to minimum $\chi^{2} / \mathrm{df}$ ( $\mathrm{df}$ is degrees of freedom) led to optimum values of P4-P6. For P5 as a starting point in optimization we took several values corresponding to $\mathrm{T}$ in interval $(8,16)$ years. For such selections the result was stable and it converged to the best frequency P5 $=0.53 \pm$ 0.03 . Figures 5 and 6 summarize the results.

Although the fit provides optimum for time of the cycle which is close to those of solar activity, geomagnetic activity and cosmic rays, it is not matching exactly the main cyclicity of $\sim 11$ years. Figure 6 presents the fitting details.

In comparison the profiles of $\chi^{2} / \mathrm{df}$ of $\mathrm{N}^{\prime}$ and SSN as a function of $\mathrm{T}_{\mathrm{per}}$, two different features are seen. First one is the different position of the best P5 fits: while for SSN it is $\sim 11$ years, for $\mathrm{N}^{\prime}$ it is $\sim 11.8$ years. The second one is the difference in profiles of $\chi^{2} / \mathrm{df}$ : for SSN the minimum is better pronounced, the decrease of $\chi^{2} / \mathrm{df}$ is by a factor of $\sim 20$, while for $\mathrm{N}^{\prime}$ it is just $\sim 1.5$ (ratio of $\chi^{2} / \mathrm{df}$ at $\mathrm{T}_{\text {per }}=8$ years to that at the optimum length of the cycles). Difference is also seen in Lomb-Scargle periodograms showing the normalized amplitude of power spectrum density as illustrated in Figure 7.

Dimitrov et al. [18] report also slightly higher cyclicity in suicide incidence rate $(12.5 \mathrm{yr})$ than that of heliophysical parameters (Figure 2 of the cited paper). Figure 7 indicates also other features in the spectrum density of $\mathrm{N}$ profiles different from those of heliophysical parameters. The "high frequency" part of the spectra in $\mathrm{N}$ is different from those of SSN, CRLS and AP'. The zoomed part of the spectra is revealing possible contributions. The highest one in the range Tper $=2$ 4 years is that with the center at $\sim 2.2$ years. Such cyclicity was reported earlier in suicides in England and Wales based on data 1968 - 2002 [19].

\section{Summary}

The yearly data of suicide events occurred in Slovakia plus Czech Republic in 1982-2011 (N) have been compared with geomagnetic
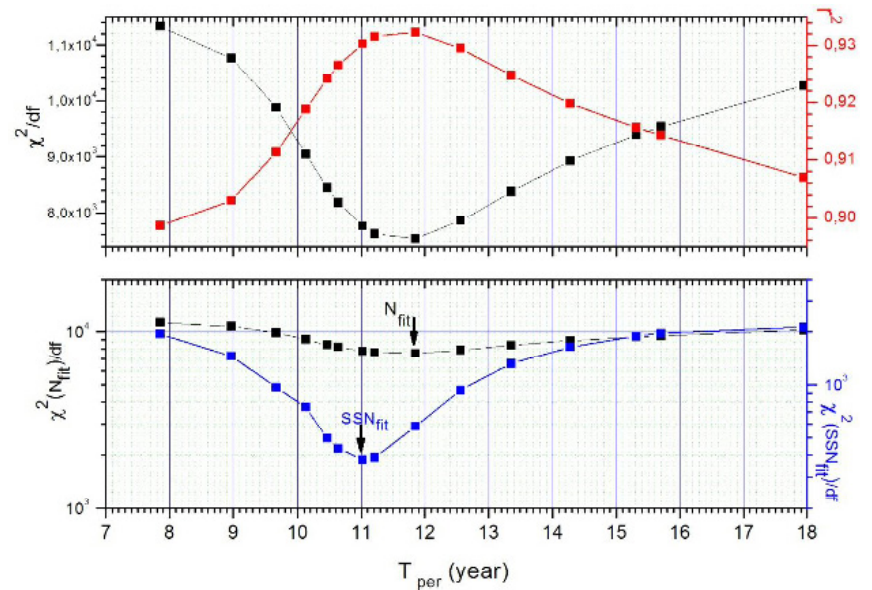

Figure 6: By fixing the values $\mathrm{P} 1-\mathrm{P} 3$ and checking $\mathrm{X}^{2} / \mathrm{df}$ as well as $\mathrm{r}^{2}$ coefficient for several P5 values fixed (corresponding to $T=2 \pi / P 5$ ), the optimum values P4 and P6, being the amplitude and phase of the cosine function, is found. The same procedure was done for the solar activity and other outer space indices. Lower panel displays the $\mathrm{X}^{2} / \mathrm{df}$ for the fits of $\mathrm{N}$ and $\mathrm{SSN}$ respectively in the logarithmic scale having the same range of values. 
Citation: Kancírová M, Kudela K (2014) The Relationship between Suicide Incidents in Slovakia and the Czech Republic and Heliophysical Parameters: Empirical Results. Astrobiol Outreach 2: 116. doi: 10.4172/2332-2519.1000116
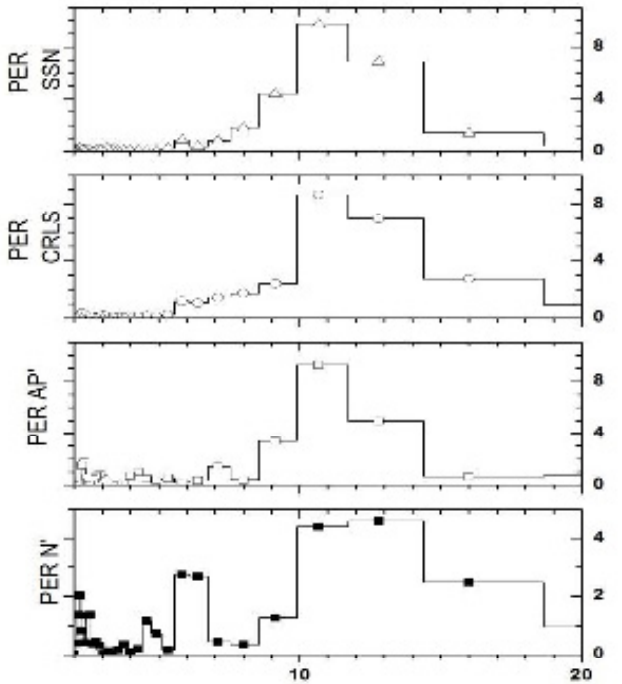

TPER (yr)

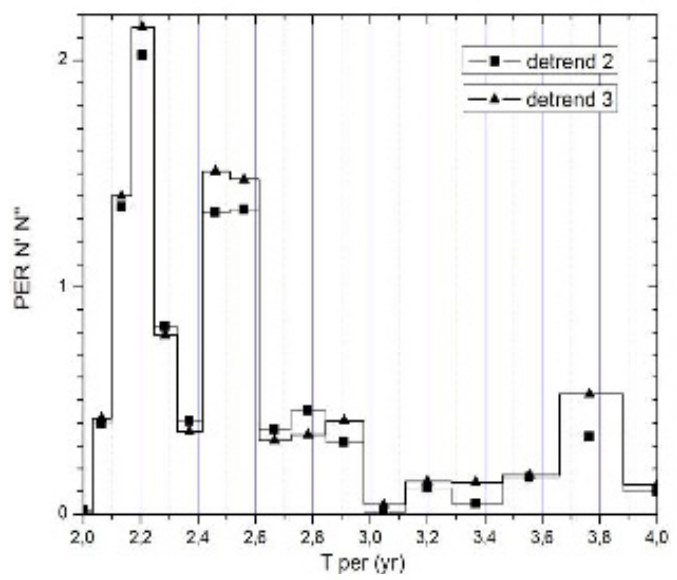

Figure 7: Periodograms (Lomb-Scargle spectrum density, points) of the values analyzed, namely SSN, CRLS, detrended AP by polynomial 2 , and detrended $\mathrm{N}$ by the same order polynom - left panel. Points are computed values. Right panel shows high frequency part of the spectrum density of $\mathrm{N}$ detrended by quadratic (2) and cubic (3) polynom. The increase at $\sim 2.2 \mathrm{yr}$ has highest significance in the $2-4$ year range of cyclicities.

(Ap), heliospheric (CRI) and solar (SSN, F10.7) activity indices. Rather weak linear relationship between heliophysical parameters and suicide incidents is found. The trends and linear correlation coefficients obtained can be marked as just based on the pairs of the data available and no causality is deduced. There may be also different factors influencing the suicide occurrence than just heliophysical ones (e.g. discussed by $[20,21]$. The results can be summarized:

1. Low number of points (30) and low statistics reveals the correlation coefficients between $\mathrm{N}$ and SSN, CRI having low statistical significance. The moderate one is found in comparison of $\mathrm{N}$ and the geomagnetic activity index Ap. Linear multiregression including all three indices provides slightly better correlations.

2. There is a long term trend persistent in the time series $\mathrm{N}(\mathrm{t})$. Low order polynomial fits in time significantly improve the correlations. Thus other, non-heliophysical influences probably play important role.
3. In detrended data $N^{\prime}$ the search of periodicities near the strongest one known in heliophysical data ( $\sim 11$ years) leads to identification of the cyclicity with the center at $\sim 11.8$ years and rather wide profile, not exactly matching the profiles of SSN, CRI and AP cyclicities.

4. Progress in the description and understanding the features in periodograms of suicide event occurrence (both spectral density and the phases) as well as in correlations with the outer space characteristics require better statistics, data from other regions (e.g. at various geomagnetic latitudes) and better temporal resolution.

\section{Acknowledgement}

The National Health Information Center Slovakia and Czech Statistical Office are acknowledged for the data on suicide incidents. Measurements of cosmic rays at Lomnický štít are supported by IEP SAS and by VEGA grant agency project 2/0040/13.

\section{References}

1. Dimitrova S, Mustafa FR, Stoilova I, Babayev ES, Kazimov EA (2009) Possible influence of solar extreme events and related geomagnetic disturbances on human cardio-vascular state: Results of collaborative Bulgarian-Azerbaijani studies. Advances in Space Research 43: 641-648

2. Mavromichalaki H, Papailiou M, Dimitrova S, Babayev ES, Mustafa FR (2008) Geomagnetic Disturbances and Cosmic Ray Variations in Relation to Human Cardio-health State: A Wide Collaboration. ECRS 2008, Kosice, 8-12 September 2008.

3. Mavromichalaki H, Papailiou M, Dimitrova S, Babayev ES, Loucas P (2012) Space weather hazard and their impact on human cardio-health state parameters on Earth. Natural Hazards 64:1447-1459.

4. Ghione S, Mezzasalma L, Del Seppia C, Papi F (1998) Do geomagnetic disturbances of solar origin affect arterial blood pressure? J Hum Hypertens 12: $749-754$

5. Verma PL (2012) Suicide incidents in India in relation with solar activity parameters and cosmic ray intensity (1989 to 2011). International Journal of Physical Sciences 7: 6240-6245.

6. Cornelissen G, Dimitrov BD, Carandente F, Halberg F (2011) Space and earth weather mirrored in patterns of suicide incidence. World Heart Journal 3: 31-42.

7. Stoupel E, Shimshoni M (1991) Hospital cardiovascular deaths and tota distribution of deaths in 180 consecutive months with different cosmic physical activity: a correlative study (1974-1988). International Journal of Biometeorology 35: 6- 9

8. Gnevyshev MN, Novikova KF (1972) The influence of solar activity on the earth's biosphere. Journal of Interdisciplinary Cycle Research 3: 99-104.

9. Stoupel E, Babayev ES, Mustafa FR, Abramson E, Israelevich P, et al (2006) Clinical Cosmobiology-Sudden Cardiac Death and Daily/Monthly Geomagnetic, Cosmic Ray and Solar Activity-the Baku Study (2003-2005). Sun and Geosphere 1: 13-16.

10. Palmer SJ, Rycroft MJ, Cermack M (2006) Solar and geomagnetic activity extremely low frequency magnetic and electric fields and human health at the Earth's surface. Survey in Geophysics 27: 557-595.

11. Gordon CH, Berk M (2003) The effect of geomagnetic storms on suicide. S Afr Psychiatry Rev 6: 24-26.

12. National Health Information Center (2006) Suicide and suicide attempts in Slovakia in 2005 .

13. National Health Information Center (2013) Suicide and suicide attempts in Slovakia in 2012.

14. Czech Statistical Office (2013) Suicides by sex and age group: 1945-2011.

15. Kenny DA (1986) Statistics for the social and behavioral sciences. Little, Brown and Company, Canada.

16. Tada H, Nishimura T, Nakatani E, Matsuda K, Teramukai S, Fukushima $M$ (2014) Association of geomagnetic disturbances and suicides in Japan 19992010. Environ. Health Prev. Med. 19: 64-71. 
Citation: Kancírová M, Kudela K (2014) The Relationship between Suicide Incidents in Slovakia and the Czech Republic and Heliophysical Parameters: Empirical Results. Astrobiol Outreach 2: 116. doi: 10.4172/2332-2519.1000116

Page 5 of 5

17. Nishimura T, Tada H, Nakatani E, Matsuda K, Teramukai S, et al. ( 2014) Stronger geomagnetic field may be a risk factor for male suicides. Psychiatry and Clinical Neurosciences 68: 404-409.

18. Dimitrov BD, Atanassova PA, Rachkova MI (2009) Cyclicity of suicides may be modulated by internal or external 11 year cycles: an example of suicide rates in Finland. Sun and Geosphere 4: 50-54

19. Cornelissen G, Halberg F (2006) Chronomics of suicides and the solar wind. The British Journal of Psychiatry 189: 567-568.
20. Shumilov OI, Kasatkina EA, Novikova TB, Sutinen ML, Chramov AV, et al (2014) Natural and man-made influences on suicides in northwestern Russia. Nat. Hazards.

21. Kasatkina EA, Shumilov O I, Novikova TB, Chramov AV (2014) Peculiaririties of Dynamics and Cyclicity of death from suicides and heliophysical and anthropological factors in norther region Kola (in Russian), Human Ecology 2: $45-50$ 